\title{
Maintenance environnementale et politique fiscale optimale dans un modèle à générations imbriquées
}

\author{
Mouez Fodha* \\ ERASME et EUREQua, Université Paris 1 Panthéon-Sorbonne**
}

\section{Introduction}

Dans les pays de l'OCDE, il est de plus en plus fréquent d'affecter les recettes des taxes environnementales à des dépenses nécessaires aux mesures de politique de l'environnement, ou de les utiliser pour créer des recettes pour des fonds ou des agences de l'environnement (OECD (2001)). En France ${ }^{1}$, les recettes de la taxe générale sur les activités polluantes (TGAP, votée en 1999) sont versées à l'ADEME et sont destinées à couvrir les dépenses à finalité environnementale. Ainsi, la plupart des initiatives visant à introduire des taxes environnementales naissent-elles de la nécessité de trouver des sources de recettes, qui puissent servir à financer des dépenses pour la sauvegarde de l'environnement, plutôt que d'une volonté de tirer partie de leurs propriétés incitatives.

Les dépenses de protection de l'environnement, nommées maintenance environnementale dans cet article, financent deux catégories d'opérations distinctes : d'une part, la dépollution, qui constitue un traitement curatif de la pollution (collecte et traitement des déchets industriels et ménagers, pose de filtres à particules en bout de chaînes...) afin que l'environnement ne se dégrade pas par suite de l'activité humaine; et d'autre part, l'entretien de

* Je tiens à remercier Philippe Michel et l'ensemble des membres du groupe de travail "Générations Imbriquées " pour leurs commentaires sur une version préliminaire de cet article. Je remercie également les deux rapporteurs anonymes pour leurs remarques constructives. Je reste seul responsable des erreurs ou omissions.

** Maison des Sciences Économiques, 106-112 Boulevard de l'Hôpital, 75647 Paris Cedex 13.

Tél. : 331440782 21. Fax: 331440782 31. email: fodha@univ-paris1.fr

1 D'après l'líen, en 2002, la dépense de protection de l'environnement représente $2 \%$ du Pib français, mais elle augmente de 5, 8\% par an en moyenne depuis 1990 (Ifen (2004)). 
l'environnement, qui correspond aux interventions permettant d'améliorer l'environnement par rapport à un état naturel (taille des arbres, lutte contre les intempéries et la sécheresse, protection d'espèces en voie d'extinction...).

L'objectif de notre article est de savoir si la maintenance publique peut être un instrument de décentralisation de l'optimum social. Nous analysons les propriétés d'affectation des recettes fiscales à un secteur public de maintenance environnementale et nous déterminons le niveau optimal de fourniture de cette activité. Nous montrons alors que l'activité de dépollution est un instrument de la politique environnementale mais que, contrairement à ce que l'on rencontre dans la littérature, il ne suffit pas. L'entretien est également nécessaire.

Les principaux travaux traitant des politiques fiscales environnementales concernent uniquement le financement d'une activité de dépollution ${ }^{2}$ et, de plus, ne cherchent pas à définir les stratégies optimales. En effet, dans un cadre statique, l'apport de la prise en compte d'une activité publique de dépollution est essentiellement de permettre un réexamen de la problématique du double dividende ${ }^{3}$ (Bovenberg et van der Ploeg (1994), Ligthart et van der Ploeg (1996)). Cette littérature s'est alors principalement focalisée sur le caractère plus ou moins distorsif des taxes environnementales par rapport à toute autre taxe $\mathrm{e}^{4}$. Par ailleurs, dans un cadre intertemporel, lorsque l'économie présente des propriétés de croissance économique potentiellement illimitée, les études se sont concentrées sur les propriétés dynamiques de l'économie planifiée. Le recours à une activité de dépollution devient alors une condition nécessaire (mais non suffisante) à l'obtention d'une durabilité de la croissance ${ }^{5}$ (van der Ploeg et Withagen (1991), van Marrewijk, van der Ploeg et Verbeek (1993), Beltratti (1995), Michel et Rotillon (1996)). Cependant, comme le montre Ragot (1997), la croissance durable n'est possible que sous des hypothèses restrictives sur les élasticités de substitution intertemporelle de la fonction d'utilité (nécessairement supérieures à l'unité) et sur la fonction de dépollution (homogène de degré zéro).

Enfin, dans le cadre des modèles à générations imbriquées, lorsque l'environnement est une externalité pure, la résolution du modèle de façon temporellement cohérente est complexe en présence de plusieurs externalités. Les travaux de Michel (1993) et Marini et Scaramozzino (1995) portent également sur la détermination de l'optimum. La généralité de ces approches ne permet pas de clarifier les modalités de mise en place de l'intervention publique. En effet, il apparait que la mise en œuvre des politiques optimales nécessite la connaissance précise des préférences des ménages et des

2 Lactivité de dépollution prend la forme soit de dépenses de dépollution, soit d'un secteur explicite de dépollution nécessitant l'allocation de ressources en capital eVou travail.

3 En substituant une taxe environnementale a une autre taxe, il y a " double dividende " si cette poitique tiscale permet d'une part, d'améliorer la qualité de l'environnement et d'autre part, de diminuer les distorsions du système fiscal.

4 II semble cependant que l'affectation des revenus de la taxe à une activité de dépollution ne lève aucune des incertitudes concernant l'occurrence du deuxième dividende.

5 Une croissance est dite « durable " si elle permet de concilier croissance économique et non dégradation de l'environnement. 
technologies de la dépollution. En revanche, lorsque les recettes pour l'environnement résulte de contributions volontaires de la part des agents, les travaux posent plus explicitement la structure de décentralisation de l'optimum (John et Pecchenino (1994), John et alii (1995), Ono (1996), Jouvet, Michet et Vidal (2000)). Mais dans ce contexte, l'environnement n'est plus une externalité pure.

Cette littérature, en ne considérant généralement que l'activité de dépollution, ne permet pas de distinguer entre les différents modes d'intervention de l'État dans la lutte contre les externalités environnementales. Dans cet article, nous montrons en revanche, que si la maintenance environnementale est un instrument d'internalisation des externalités, sa forme ne peut se limiter à l'activité de dépollution. Pour ce faire, nous examinons précisément les modalités de décentralisation de l'intervention publique dans le cadre d'une économie fermée, à générations imbriquées non-altruistes, et confrontées à deux sources dynamiques d'inefficacités. D'une part, les émissions de polluants issus de la consommation affectent le bien-être des générations suivantes et sont donc à l'origine d'une externalité intergénérationnelle; d'autre part, le recyclage spécifique des recettes fiscales, permettant de financer l'activité de maintenance environnementale, n'est pas pris en compte par les agents lors de leur procédure d'arbitrage (inefficacité intragénérationnelle liée au problème de fourniture du bien public). On montre que l'équilibre concurrentiel n'est pas optimal mais que la maintenance environnementale est un instrument à la disposition du gouvernement qui, associé à une structure fiscale bien précise, permet de décentraliser l'optimum. Cette structure fiscale corrige donc l'externalité de pollution, mais également la distorsion sur le marché du capital, induit par l'égoïsme des individus, ainsi que l'inefficacité intragénérationnelle découlant du caractère public de la maintenance environnementale.

La section 1 présente les hypothèses de l'économie concurrentielle. La section 2 détermine l'optimum social escompté. La troisième section fixe le niveau optimal des taux de taxes permettant de décentraliser l'optimum. Enfin, la quatrième section montre, qu'avec les spécifications retenues, la maintenance environnementale publique ne peut se limiter à l'activité de dépollution mais doit faire intervenir une nouvelle composante, l'entretien, souvent négligée par la littérature.

\section{$1 \quad$ L'économie concurrentielle}

On suppose qu'à chaque période naît un agent représentatif vivant deux périodes. À sa première période de vie, l'agent jeune $(j)$ travaille (l'offre de travail est inélastique et est égale à l'unité), perçoit un salaire $w_{t}$ avec lequel il épargne $s_{t}$, consomme $c_{t}$ et paie à un gouvernement une taxe forfaitaire $T_{t} . \tau_{t}^{c}$ et $\tau_{t}^{k}$ sont respectivement les taux de taxe sur la consommation et sur les rendements de l'épargne. Lorsqu'il est à la retraite, l'individu vieux 
(v) consomme $d_{t+1}$ représentant l'intégralité de son épargne rémunérée au facteur d'intérêt $1+r_{t+1}$. Le taux d'escompte intertemporel est $\frac{\beta}{1-\beta}$ avec $0<\beta<1$. L'agent est en outre affecté positivement par le niveau de la qualité environnementale $Q_{t}$, la sensibilité à la qualité de l'environnement est représentée par les termes $\phi_{i} \in[0,1], i=j, v$. L'environnement est une externalité pour l'agent.

Le programme d'un agent né en $t$ s'écrit :

$\operatorname{Max}$ $c_{t}, d_{t+1}$

$$
U\left(c_{t}, Q_{t}, d_{t+1}, Q_{t+1}\right)=u\left(c_{t}\right)+\phi_{j} z\left(Q_{t}\right)+\beta\left[v\left(d_{t+1}\right)+\phi_{v} g\left(Q_{t+1}\right)\right]
$$

sous les contraintes budgétaires :

$$
\left\{\begin{array}{l}
w_{t}=\left(1+\tau_{t}^{c}\right) c_{t}+s_{t}+T_{t} \\
\left(1+\tau_{t+1}^{c}\right) d_{t+1}=\left(1+r_{t+1}\right)\left(1-\tau_{t}^{k}\right) s_{t}
\end{array}\right.
$$

Les conditions nécessaires du premier ordre déterminent la règle d'arbitrage entre consommations présente et future:

$$
\frac{u_{c}^{\prime}}{\left(1+\tau_{t}^{c}\right)}-\beta\left(1+r_{t+1}\right) \frac{\left(1-\tau_{t}^{k}\right)}{\left(1+\tau_{t+1}^{c}\right)} v_{d}^{\prime}=0
$$

Il existe dans l'économie une firme représentative maximisant son profit à chaque période. L'entreprise est soumise aux règles de la concurrence parfaite, et produit à chaque date, un bien unique en quantité $Y_{t}$. Les conditions technologiques de production satisfont les conditions d'Inada et sont représentées par $Y_{t}=F\left(K_{t}, N\right)$, où $F($.$) admet des rendements d'échelle$ constants, que l'on réécrit en grandeurs par tête de jeunes en $t$ :

$$
y_{t}=f\left(k_{t}\right) \quad f^{\prime}>0 ; \quad f^{\prime \prime} \leqslant 0
$$

avec $y=\frac{Y}{N}$ et $k=\frac{K}{N}$.

On suppose qu'il y a dépréciation totale du stock de capital en une période et que le bien privé représente le numéraire. Les conditions du premier ordre du programme de maximisation du profit égalisent la productivité marginale des facteurs à leurs coûts réels et sont données par :

$$
\left\{\begin{array}{l}
f^{\prime}\left(k_{t}\right)=1+r_{t} \\
f\left(k_{t}\right)-k_{t} f^{\prime}\left(k_{t}\right)=w_{t}
\end{array}\right.
$$

La qualité de l'environnement est un bien public dont la maintenance relève exclusivement de l'État. Le gouvernement se charge de prélever les impôts sur les ménages, dont une partie $G$ est consacrée à l'amélioration 
de la qualité de l'environnement. Ces recettes $G$ sont donc transformées en activités vertes, la maintenance, en intervenant directement sur la qualité de l'environnement. Cette dernière est mesurée par un indice agrégé $Q_{t}$ qui représente la valeur des aménités provenant de la qualité environnementale. La dynamique de l'indice de qualité environnementale est régit par la loi d'évolution suivante:

$$
Q_{t+1}=[1-h] Q_{t}-\alpha_{j} c_{t}-\alpha_{v} d_{t}+\mu G_{t}
$$

où $G_{t}$ est le montant de l'engagement public pour la maintenance environnementale (son rendement marginal est $\mu>0) ; h \in[0,1]$ représente le taux de variation autonome de l'environnement et $\alpha_{i} \geqslant 0(i=j, v)$ le taux d'émission de pollution issue de la consommation ${ }^{6}$ des agents. Ainsi, l'indice reflète par exemple la qualité des parcs publics, de forêts, de sentiers de promenade ou de randonnées. Sans intervention de l'activité humaine, cette qualité a naturellement tendance à se dégrader, l'indice tend alors à être nul. Le terme $h$ s'interprète comme la vitesse de dégradation naturelle de l'environnement.

L'État dispose d'une caisse équilibrée à toutes les périodes. Elle prend en compte les transferts forfaitaires, la taxe sur les rendements du capital et la taxe sur la consommation des agents. On a donc:

$$
G_{t}=T_{t}+\tau_{t}^{k}\left(1+r_{t}\right) s_{t-1}+\tau_{t}^{c}\left(c_{t}+d_{t}\right)
$$

Un équilibre de laissez-faire de notre économie est défini par une séquence de décisions, de prix et de taxes

$\left\{y_{t}, G_{t}, k_{t}, c_{t}, d_{t}, s_{t}, w_{t}, r_{t}, T_{t}, \tau_{t}^{k}, \tau_{t}^{c}\right\}_{t=1}^{\infty}$ telle que, à chaque date $t=1,2 \ldots$ :

(i) les agents maximisent (1) sous les contraintes (2);

(ii) la firme maximise son profit;

(iii) les marchés sont équilibrés (capital, travail, biens);

(iv) l'indice de qualité environnementale évolue selon sa loi (6); et $\left\{k_{0}, Q_{0}\right\}$ données.

À l'équilibre général, la loi de Walras nous permet de ne retenir que l'équilibre sur le marché du capital, qui s'écrit:

$$
k_{t+1}=s_{t}
$$

avec $s_{t}$ la fonction d'épargne ${ }^{7}$ de la génération née en $t$. L'équilibre stationnaire est tel que $k_{t+1}=k_{t}=k^{*}$.

6 il n'existe pas de motif a priori de distinguer $\alpha_{j}$ de $\alpha_{v}$. Nous faisons néanmoins cette hypothèse atin d'introduire les externalités intergénérationelles de consommation telles qu'elles se trouvent dans une grande partie de la littérature (John et Pecchenino (1994), John et atii (1995), Ono (1996), Yoshida (2002),...).

7 Une analyse détaillée de la fonction d'épargne se trouve dans de la Croix et Michel (2002). 


\section{$2 \quad$ Optimum de long terme}

On suppose l'existence d'un planificateur cherchant à maximiser l'utilité de l'ensemble des générations, escomptée par un facteur d'actualisation social, noté $\theta(0<\theta<1)$. On associe à la contrainte de ressources de la période $t$ le multiplicateur $\theta^{t} \lambda_{1, t}$; de même, à la contrainte d'évolution de $Q_{t}$ on associe le multiplicateur $\theta^{t} \lambda_{2, t}$. $k_{0}$ et $Q_{0}$ sont données.

Le lagrangien associé au programme du planificateur s'écrit:

$$
\begin{aligned}
& L_{t}\left(c_{t-1}, d_{t}, k_{t}, Q_{t-1}, G_{t-1}\right)= \\
& \sum_{t=1}^{+\infty} \theta^{t-1}\left[u\left(c_{t-1}\right)+\phi_{j} z\left([1-h] Q_{t-2}-\left(\alpha_{j} c_{t-2}+\alpha_{v} d_{t-2}\right)+\mu G_{t-2}\right)\right. \\
& \left.+\beta v\left(d_{t}\right)+\beta \phi_{v} g\left([1-h] Q_{t-1}-\left(\alpha_{j} c_{t-1}+\alpha_{v} d_{t-1}\right)+\mu G_{t-1}\right)\right] \\
& -\sum_{t=1}^{+\infty} \theta^{t-1} \lambda_{1, t-1}\left[k_{t}-f\left(k_{t-1}\right)+c_{t-1}+d_{t-1}+G_{t-1}\right] \\
& \quad-\sum_{t=1}^{+\infty} \theta^{t-1} \lambda_{2, t-1}\left[Q_{t-1}-[1-h] Q_{t-2}+\left(\alpha_{j} c_{t-2}+\alpha_{v} d_{t-2}\right)-\mu G_{t-2}\right]
\end{aligned}
$$

Les conditions nécessaires d'optimalité sont, à l'état stationnaire ${ }^{8}$ :

$$
\begin{gathered}
\frac{\partial L_{t}(.)}{\partial c_{t-1}}=u^{\prime}-\theta \alpha_{j} \phi_{j} z^{\prime}-\beta \alpha_{j} \phi_{v} g^{\prime}-\lambda_{1}-\lambda_{2} \theta \alpha_{j}=0 \\
\frac{\partial L_{t}(.)}{\partial d_{t}}=\frac{\beta}{\theta} v^{\prime}-\theta \alpha_{v} \phi_{j} z^{\prime}-\beta \alpha_{v} \phi_{v} g^{\prime}-\lambda_{1}-\lambda_{2} \theta \alpha_{v}=0 \\
\frac{\partial L_{t}(.)}{\partial k_{t}}=-\lambda_{1}+\lambda_{1} \theta f_{k}^{\prime}=0 \\
\frac{\partial L_{t}(.)}{\partial G_{t-1}}=\theta \mu \phi_{j} z^{\prime}+\beta \mu \phi_{v} g^{\prime}-\lambda_{1}+\lambda_{2} \theta \mu=0 \\
\frac{\partial L_{t}(.)}{\partial Q_{t-1}}=\theta(1-h) \phi_{j} z^{\prime}+\beta(1-h) \phi_{v} g^{\prime}-\lambda_{2}+\lambda_{2} \theta(1-h)=0
\end{gathered}
$$

La condition (10) fixe le stock de capital à l'optimum $\hat{k}$ au niveau de la règle d'or modifiée :

$$
f^{\prime}(\hat{k})=\frac{1}{\theta}
$$

La relation (12) nous donne le prix implicite de la qualité environnementale:

$$
\lambda_{2}=\frac{1-h}{\frac{1}{\theta}-1+h}\left[\phi_{j} z^{\prime}+\frac{\beta}{\theta} \phi_{v} g^{\prime}\right] \geqslant 0
$$

\footnotetext{
8 Nous nous intéressons uniquement aux solutions intérieures.
} 
Ce prix implicite, qui s'interprète comme le bénéfice social marginal de la variation de la qualité de l'environnement, doit être égal à la valeur escomptée de l'utilité marginale de l'environnement.

De façon symétrique, les conditions (11) et (13) fixent le prix implicite du capital :

$$
\lambda_{1}=\frac{\mu}{1-h} \lambda_{2} \geqslant 0
$$

Ce prix implicite du capital est donc égal à l'impact marginal, évalué en terme de bien-être, de la maintenance publique $G$ sur la qualité environnementale. On montre également que ce prix implicite doit être égal à l'utilité marginale de la consommation. En effet, les conditions (8), (9) et (13) nous donnent la règle d'allocation intertemporelle de la consommation, qui égalise utilité marginale d'un agent jeune à celle escomptée d'un agent vieux:

$$
\begin{aligned}
& u^{\prime}-\frac{\alpha_{j}}{(1-\theta(1-h))}\left[\theta \phi_{j} z^{\prime}+\beta \phi_{v} g^{\prime}\right]= \\
& \frac{\beta}{\theta} v^{\prime}-\frac{\alpha_{v}}{(1-\theta(1-h))}\left[\theta \phi_{j} z^{\prime}+\beta \phi_{v} g^{\prime}\right]=\lambda_{1}
\end{aligned}
$$

L'utilité marginale de la consommation admet deux dimensions : la première est le gain en bien-être issu de la consommation, alors que la deuxième mesure la perte en bien-être engendrée par les émissions de déchets issus de l'activité de consommation.

Le niveau optimal de la consommation prend ainsi en compte les conséquences sur la pollution pour les générations futures; cet impact de long terme $(1-h)$ est pondéré par le facteur d'escompte social $\theta$. publique :

Les relations (14) et (15) déterminent l'offre optimale de maintenance

$$
\mu \frac{\phi_{j} z^{\prime}+\frac{\beta}{\theta} \phi_{v} g^{\prime}}{\frac{1}{\theta}-1+h}=\frac{u^{\prime}}{1+\frac{\alpha_{j}}{\mu}}=\frac{\beta}{\theta} \frac{v^{\prime}}{1+\frac{\alpha_{v}}{\mu}}
$$

Le niveau optimal de maintenance est tel que l'utilité marginale de la consommation polluante soit égale au rendement marginal de la maintenance. Ce niveau est donc défini par la règle de Samuelson de fourniture d'un bien public (Samuelson (1954)). Notons que le rendement marginal de la maintenance publique admet deux composantes, la première étant l'effet direct de la maintenance sur le bien-être environnemental $(\mu)$. La deuxième composante mesure l'effet indirect de la maintenance sur le bien-être environnemental transitant par la baisse de la consommation polluante $\left(\alpha_{j}\right.$ et $\alpha_{v}$ ): plus le planificateur affecte de la ressource à la maintenance et plus il diminue le niveau de consommation.

Sous cette condition (16), le planificateur internalise l'externalité négative de la consommation (dégradation la qualité environnementale future) 
et la présence de l'activité publique de maintenance (amélioration de la qualité environnementale future). Nous retrouvons ainsi le taux d'escompte pertinent pour l'évaluation des politiques de l'environnement défini par Marini et Scaramozzino (1995), égal à la somme du taux d'escompte social pur ${ }^{9} \delta$ et du taux d'assimilation naturelle $h$.

Le niveau optimal de l'activité de dépollution est égal à l'évaluation, en termes de bien-être, du dommage marginal de l'externalité négative de la consommation, définie par :

$$
\left(z^{\prime} \phi_{j}+\frac{\beta}{\theta} g^{\prime} \phi_{v}\right) \frac{\alpha_{j}+\alpha_{v}}{\frac{1}{\theta}-(1-h)}
$$

Cette activité de dépollution n'est qu'une des deux composantes de l'intervention publique de maintenance. En effet, on montre que si le planificateur impose une maintenance strictement égale au niveau optimal de la dépollution, il ne fait qu'absorber exactement le montant de pollution. Mais dans ce cas, seule l'externalité négative de consommation est corrigée et, à long terme, la qualité environnementale tend à être nulle ${ }^{10}$.

En revanche, avec la règle (16) d'affectation des ressources, on montre que la qualité environnementale, à l'optimum, est toujours positive. La structure optimale de la maintenance doit respecter la règle suivante : elle doit prendre en compte simultanément l'activité de dépollution à son niveau optimal et l'utilité marginale de la maintenance dont l'objectif est une meilleure qualité environnementale à lung terme $(\hat{Q}>0)$. Cette dernière intervention correspond à une activité d'entretien de l'environnement.

Là règle de distribution intergénérationnelle au niveau de maintenance optimale est déterminée par la condition (16) qui s'écrit également:

$$
\frac{u^{\prime}}{v^{\prime}}=\frac{\beta}{\theta} \frac{1+\frac{\alpha_{j}}{\mu}}{1+\frac{\alpha_{v}}{\mu}}
$$

Notre système d'équations (8) à (12) nous permet de déterminer $\{\hat{c}, \hat{d}, \hat{k}, \hat{G}, \hat{Q}\}$ :

$$
\mid \begin{gathered}
\frac{u^{\prime}}{\theta \phi_{j} z^{\prime}+\beta \phi_{v} g^{\prime}}=\frac{\mu+\alpha_{j}}{1-\theta(1-h)} \\
\frac{\beta}{\theta} \frac{v^{\prime}}{\theta \phi_{j} z^{\prime}+\beta \phi_{v} g^{\prime}}=\frac{\mu+\alpha_{v}}{1-\theta(1-h)} \\
f^{\prime}(\hat{k})=\frac{1}{\theta} \\
f(\hat{k})-\hat{k}=\hat{c}+\hat{d}+\hat{G} \\
\hat{Q}=\frac{-\left(\alpha_{j} \hat{c}+\alpha_{v} \hat{d}\right)+\mu \hat{G}}{h}
\end{gathered}
$$

\footnotetext{
9 Le taux d'escompte social pur est défini par $\delta=\frac{1}{\partial}-1$.

10 Remarquons que si $\mu G=\alpha_{j} c+\alpha_{v} d$, nous avons alors, à long terme, $Q$ qui tend à ètre nul. Les générations futures, dans ce cas, sont lésées et ne retirent aucune satisfaction de la qualité environnementale.
} 


\section{Décentralisation}

Nous cherchons les taux de taxes que doit proposer un planificateur au gouvernement de telle sorte que le programme de ce dernier à l'équilibre concurrentiel coïncide avec l'optimum de long terme. Les instruments à la disposition de l'agent régulateur sont ceux présentés dans le cadre de l'économie concurrentielle. En présence de trois sources d'inefficacités économiques (sur ou sous-accumulation du capital - consommations polluantes fourniture d'un bien public) et donc de trois cibles, la structure de taxestransferts se compose de trois instruments $\left\{\hat{T}, \hat{\tau}^{k}, \hat{\tau}^{c}\right\}$; elle doit permettre de retrouver l'intensité capitalistique définie par la règle d'or, internaliser les flux de polluants issus de la consommation (externalité négative) et enfin entretenir une activité de maintenance.

Afin de décentraliser l'optimum, on suppose que l'État connaît le niveau total des prélèvements pour financer la maintenance optimale $(\hat{G})$. C'est le niveau qui, d'une part, internalise les effets de la consommation sur la pollution et qui d'autre part, permet d'atteindre une qualité environnementale meilleure qu'une tendance naturelle, étant donné les technologies disponibles.

À l'état stationnaire, l'économie concurrentielle définie à la section 1 se résume par:

$$
\mid \begin{aligned}
& f_{k}^{\prime}=1+r^{*} \\
& f\left(k^{*}\right)-k^{*} f_{k}^{\prime}=w^{*} \\
& w^{*}=\left(1+\hat{\tau}^{c}\right) c^{*}+s^{*}+\hat{T} \\
& \left(1+\hat{\tau}^{c}\right) d^{*}=\left(1-\hat{\tau}^{k}\right)\left(1+r^{*}\right) s^{*} \\
& k^{*}=s^{*} \\
& \frac{v_{d}^{\prime}}{u_{c}^{\prime}}=\frac{1}{\beta\left(1+r^{*}\right)\left(1-\hat{\tau}^{k}\right)} \\
& \hat{\tau}^{k}\left(1+r^{*}\right) s^{*}+\hat{T}+\hat{\tau}^{c}\left(c^{*}+d^{*}\right)=\hat{G} \\
& Q^{*}=\frac{\mu \hat{G}-\alpha_{j} c^{*}-\alpha_{v} d^{*}}{h}
\end{aligned}
$$

Pour atteindre l'optimum, il faut que l'on retrouve la règle d'arbitrage intergénérationnelle (18) à partir de la condition d'arbitrage (3) évaluée à l'état stationnaire :

$$
\frac{1}{\beta\left(1+r^{*}\right)\left(1-\hat{\tau}^{k}\right)}=\frac{\theta}{\beta}\left(\frac{1+\frac{\alpha_{y}}{\mu}}{1+\frac{\alpha_{j}}{\mu}}\right)
$$

Dans ce cas, puisqu'à l'optimum $\left(1+r^{*}\right)=\frac{1}{\theta}$, on obtient la valeur optimale suivante du taux de la taxe:

$$
\hat{\tau}_{k}=\frac{\alpha_{v}-\alpha_{j}}{\mu+\alpha_{v}}
$$


Le rôle de la taxe $\tau^{k}$ est de modifier le rendement net de l'épargne, ce qui joue directement sur le revenu des agents vieux et ainsi, sur la consommation de deuxième période. Cette propriété explique la forme de la taxe : si $\alpha_{v}>\alpha_{j}$, la consommation des vieux est plus polluante, il est préférable de consommer étant jeune, $\hat{\tau}^{k}$ doit être positive; si $\alpha_{j}>\alpha_{v}$, la taxe doit inciter à favoriser le report de consommation, $\hat{\tau}^{k}$ est négatif. Par ailleurs, lorsque l'externalité de pollution provenant de la consommation des générations jeunes est strictement égale (en taux d'émission) à celle provenant des générations retraitées $\alpha_{j}=\alpha_{v}$; la taxe sur les rendements de l'épargne est nulle. L'État a donc besoin d'un instrument en moins si l'externalité négative de la consommation est engendrée de façon identique par l'ensemble des générations. La structure optimale de la fiscalité est similaire au cas sans émissions de pollution de la consommation. Ceci revient au cas où $\alpha_{j}=\alpha_{v}=0$ (l'externalité de pollution disparait), la taxe «optimale » associée est donc nulle $\left(\hat{\tau}_{k}=0\right)$. Il est néanmoins nécessaire de garder un certain niveau de maintenance environnementale $\hat{G}>0$, financé par les autres taxes. Ces dépenses correspondent bien à de l'activité d'entretien de l'environnement.

L'équilibre du marché du capital fixe le niveau de la taxe forfaitaire $\hat{T}$ permettant d'atteindre la règle d'or modifiée $\hat{k}$ :

$$
\hat{T} / \hat{k}=k^{*}=s\left(w\left(k^{*}\right)-\hat{T}, r\left(k^{*}\right)\right)
$$

Enfin, la taxe sur les consommations permet d'atteindre le niveau optimal de la maintenance publique $\hat{G}$ :

$$
\hat{\tau}_{k}\left(1+r^{*}\right) s^{*}+\hat{T}+\hat{\tau}^{c}\left(c^{*}+d^{*}\right)=\hat{G}
$$

on obtient, après substitution :

$$
\hat{\tau}^{c}=\frac{\hat{G}-\hat{T}-\hat{\tau}_{k} \frac{k^{*}}{\theta}}{f\left(k^{*}\right)-k^{*}-\hat{G}}
$$

\section{Application}

Nous considérons une fonction d'utilité logarithmique linéairement séparable en ses arguments:

$$
U\left(c_{t}, Q_{t}, d_{t+1}, Q_{t+1}\right)=\ln c_{t}+\phi_{j} \ln Q_{t}+\beta \ln d_{t+1}+\beta \phi_{v} \ln Q_{t+1}
$$


En ce qui concerne l'optimum, la résolution du système (19) nous donne les résultats suivants ${ }^{11}$ :

$$
\begin{aligned}
& \left\{\begin{array}{l}
\hat{G}=A\left\{\frac{\frac{\alpha_{v}}{\mu}}{1+\frac{\alpha_{v}}{\mu}}+\frac{\theta}{\beta} \frac{\frac{\alpha_{j}}{\mu}}{1+\frac{\alpha_{j}}{\mu}}+\frac{\left(\phi_{v}+\frac{\theta}{\beta} \phi_{j}\right) h}{\frac{1}{\theta}-1+h}\right\} \\
\hat{c}=\frac{\theta}{\beta} \frac{A}{1+\frac{\alpha_{j}}{\mu}} \\
\hat{d}=\frac{A}{1+\frac{\alpha_{v}}{\mu}} \\
\hat{Q}=A \frac{\left(\phi_{v}+\frac{\theta}{\beta} \phi_{j}\right) \mu}{\frac{1}{\theta}-1+h} \\
f^{\prime}(\hat{k})=\frac{1}{\theta}
\end{array}\right. \\
& \operatorname{avec} A=\frac{f(\hat{k})-\hat{k}}{1+\frac{\theta}{\beta}+\frac{\left(\phi_{v}+\frac{\theta}{\beta} \phi_{j}\right) h}{\frac{1}{\theta}-1+h}}
\end{aligned}
$$

La maintenance optimale se décompose en deux parties :

$$
\hat{G}=\underbrace{\frac{\alpha_{j}}{\mu} \hat{c}+\frac{\alpha_{v}}{\mu} \hat{d}}_{\text {dépollution }}+\underbrace{A \frac{\left(\phi_{v}+\frac{\theta}{\beta} \phi_{j}\right) h}{\frac{1}{\theta}-1+h}}_{\text {entretien }}
$$

Ainsi, si un niveau de maintenance est défini de manière à ce que le deuxième terme de la relation (22) soit nul, dans ce cas $\hat{G}$ n'est destiné qu'à l'activité de dépollution (collecte et traitement des pollutions issues de la consommation). Ce niveau permet alors d'internaliser la totalité des externalités négatives de la consommation : la qualité environnementale est nulle à long terme. Par conséquent, si l'on considère non pas un indice de qualité environnementale, mais le stock de pollution $P$, ce niveau de dépollution est celui qui mènerait au paradis écologique défini par Michel (1993).

Remarquons enfin que la qualité environnementale à l'optimum ne dépend pas des taux d'émissions de polluants $\alpha_{i}$ (les flux de polluants sont totalement absorbés) mais uniquement des termes de préférences pour l'environnement $\phi_{i}$.

11 En l'absence d'externalité $\left(\alpha_{i}=\phi_{i}=0\right)$, on retrouve l'économie à la Diamond (1965) : $\hat{c}=\frac{f(\hat{k})-\hat{k}}{1+\frac{\theta}{\beta}} \frac{\theta}{\beta}$. $\hat{d}=\frac{f(\hat{k})-\hat{k}}{1+\frac{\theta}{\beta}}, Q=G=0, f^{\prime}(\hat{k})=\frac{1}{\theta}$. 


\section{Conclusion}

La prise en charge de l'activité de maintenance environnementale par l'État doit satisfaire au critère de la fourniture optimale d'un bien public défini par la règle de Samuelson. Cependant, lorsque l'économie est caractérisée par différentes générations coexistantes, ces dernières étant à la source d'externalités intergénérationnelles, cette règle est modifiée par la prise en compte d'un taux d'actualisation social pertinent, intégrant notamment le taux d'assimilation naturelle.

En outre, si l'on considère non pas l'évolution du stock de pollution mais celle d'un indice de qualité environnementale, avec les spécifications habituellement retenues, nous ne pouvons définir de règle simple de taxation, se limitant à financer une activité de traitement curatif de la pollution. En effet, si tel était le cas, la qualité environnementale serait nulle à long terme. L'engagement public ne doit donc pas se limiter à neutraliser les émissions de polluants mais véritablement à entretenir l'environnement. La valeur de l'aménité provenant d'un parc national ou de sentiers de randonnées se dégrade si ces derniers ne sont pas entretenus mais laissés à leur état sauvage. Enfin, lorsque les taux d'émissions de polluants des consommations des générations jeunes et vieilles sont égaux, le gouvernement a besoin d'une taxe en moins pour décentraliser l'optimum, malgré les externalités de pollution. Dans ce cas de figure, la structure fiscale optimale est identique au cas où la consommation devient propre.

\section{Bibliographie}

Beltratti A. (1995), "Growth with Natural and Environmental Resources", Document de Travail E.E.E., ${ }^{\circ} 58.95$, Fondazione Eni Enrico Mattei.

Bovenberg A.L. et F. van der Ploeg (1994), "Environmental Policy, Public Finance and the Labour Market in a Second-best World", Journal of Public Economics, Vol. 55, pp. 349-390.

de la Croix D. et P. Michel (2002), A theory of Economic Growth, Cambridge University Press.

Diamond P.A. (1965), "National Debt in a neoclassical growth model", American Economic Review, Vol. 55, pp. 1126-1150.

Ifen (2004), Rapport annuel de la Commission des comptes et de l'économie de l'environnement, mars.

John A. et R. Pecchenino (1994), "An Overlapping Generations Model of Growth and the Environment", The Economic Journal, 104, pp. 13931410.

John A., R. Pecchenino, D. Schimmelpfennig et S. Schreft (1995), "Shortlived Agents and the Long-lived Environment", Journal of Public Economics, 58, pp. 127-141. 
Jouvet P.A., P. Michel et J.P. Vidal (2000), "Intergenerational Altruism and the Environment", Scandinavian Journal of Economics, vol. 102(1), pp. 135-150.

Ligthart J.E. et F. van der Ploeg (1996), "Optimal Government Policy, the Environment, Employment, and Tax Shifting", in C. Carraro et D. Siniscalco (éds.), Environmental Fiscal Reform and Unemployment, Kluwer Academic Publishers, pp. 93-120.

Marini G. et P. Scaramozzinno (1995), "Overlapping Generations and Environmental Control", Journal of Environmental Economics and Management, 29, pp. 64-77.

van Marrewijk C., F. van der Ploeg et J. Verbeek (1993), "Is Growth Bad for the Environment", Banque Mondiale, Document de Travail, $\mathrm{n}^{\circ} 1151$.

Michel P. (1993), Pollution and Growth towards the Ecological Paradise, mimeo, Université Paris I.

Michel P. et G. Rotillon (1996): "Disutility of pollution and Endogenous Growth, Environmental and Resource Economics", Vol. 6, pp. 279300.

Oecd (2001), Environmentally Related Taxes in OECD Countries : Issues and Strategies, Oecd, Paris.

Ono T. (1996), "Optimal tax schemes and the environmental externality", Economics Letters, ${ }^{\circ} 53$, pp. 283-289.

van der Ploeg F.et C. Withagen (1991), "Pollution Control and the Ramsey Problem", Environmental and Resource Economics, n ${ }^{\circ}$, pp. 215-236.

Ragot L. (1997), Croissance économique durable et pollution : un essai d'interprétation formalisée, Thèse de Doctorat, Université Paris I.

Samuelson P.A. (1954), "The Pure Theory of Public Expenditure", Review of Economics and Statistics, $\mathrm{n}^{\circ} 36$, pp. 387-389.

Yoshida M. (2002), "Intergenerational Pigouvian Tax Systems", The Japanese Economic Review, vol. 53, n², pp. 199-210. 
, 